\title{
Erratum to: Laparoendoscopic single-site (LESS) varicocelectomy with reusable components: comparison with the conventional laparoscopic technique
}

Frank Friedersdorff • Seven Johannes Aghdassi - Peter Werthemann • Hannes Cash - Irena Goranova · Jonas Felix Busch · Jan Ebbing •

Stefan Hinz $\cdot$ Kurt Miller $\cdot$ Joerg Neymeyer $\cdot$ Tom Florian Fuller

Published online: 30 April 2013

(C) Springer Science+Business Media New York 2013

\section{Erratum to: Surg Endosc}

DOI 10.1007/s00464-013-2938-z

Reference 6 should read:

Lee SW, Lee JY, Kim KH, Ha US (2012) Laparoendoscopic single-site surgery versus conventional laparoscopic varicocele ligation in men with palpable varicocele: a randomized, clinical study. Surg Endosc 26:1056-1062.

The online version of the original article can be found under doi:10.1007/s00464-013-2938-z.

F. Friedersdorff · S. J. Aghdassi · P. Werthemann - H. Cash .

I. Goranova - J. F. Busch · J. Ebbing $\cdot$ S. Hinz $\cdot$ K. Miller ·

J. Neymeyer · T. F. Fuller $(\square)$

Department of Urology, Charité Universitätsmedizin Berlin,

Charitéplatz 1, 10117 Berlin, Germany

e-mail: florian.fuller@charite.de 